\section{Shifting mosaic}

\author{
Peter D. Moore
}

Dynamic Biogeography. By R. Hengeveld. Cambridge University Press: 1990. Pp. 249. £30, \$54.50.

"DYNAMIC" is an interesting word to use in the title of a book. It suggests that the subject may well be perceived by the potential reader as static, if not stagnant, and that the author intends to dispel such an illusion. This is partially true of biogeography. There was certainly a time, perhaps just two decades ago, when biogeography was an essentially descriptive subject, being concerned mainly with the classification of distribution patterns of species and communities, but with little emphasis on the processes involved in the establishment of pattern. But this has changed in more recent times, and one might reasonably expect any modern text on biogeography to be "dynamic".

It is the insertion of the time element that converts a static approach into a dynamic one, and this must be the first test of the claim inherent in the title. Hengeveld certainly passes this test as he sets out to examine both temporal and spatial variation in biogeographical patterns and to explain underlying causes, emphasizing climatic and human induced factors. One of the main problems of biogeography, however, is what Hengeveld terms the "idiosyncratic properties of species distributions". It is difficult to make general statements on the basis of the wide range of patterns and the spread of causative factors found among plant and animal species. The detection of pattern within the patterns clearly demands sophisticated analytical techniques, and the first part of the book is concerned with the application of multivariate statistics to the classification and ordination of speciesdistribution data. This initial process in biogeographical methodology provides, in Hengeveld's view, an efficient system for hypothesis generation, such hypotheses usually relating either to the past history of the taxa, or to their ecological demands and limitations. But the two types cannot be clearly separated, for the physical and biological environment is itself not static, so ecological demands simply place limits on species distribution within the ever-rolling historical framework.

The logical progression of this line of thought would take us into a consideration of changes in the ranges of selected taxa

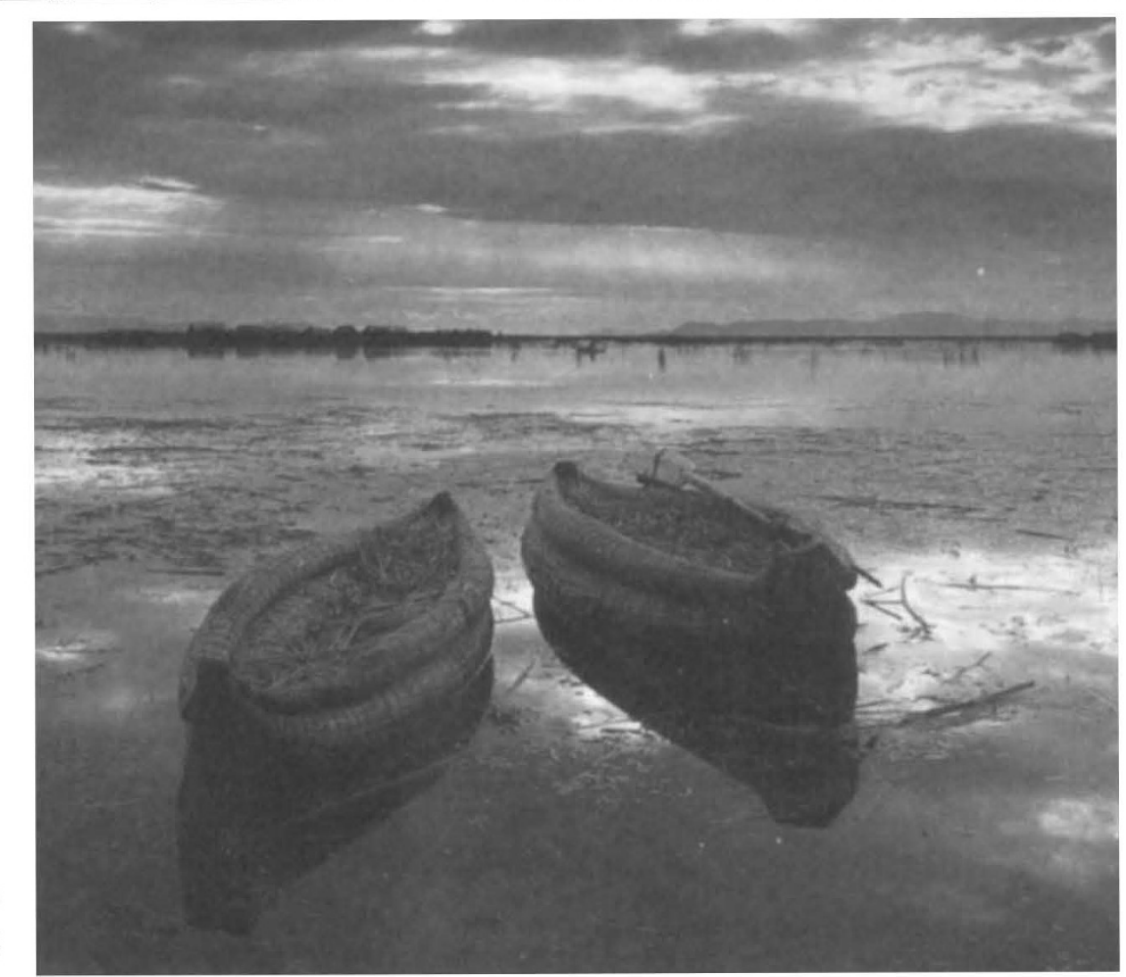

Whatever you do, wherever you go, plants will have made some contribution - this is the message of Plants for People by Anna Lewington. The volume presents both well known and not so well known uses of plants; there are details of those that protect, cure, transport (reed boats above), entertain and the better known examples of those that clothe and feed us. The book, well researched and presented, illustrates almost all examples in full colour. Interesting information gleaned from the pages includes the story of the fine cloth made from pineapple leaves, the bamboo used for scaffolding and the anaesthetics from the Andes. Published by The Natural History Museum, London. Price is $£ 19.95$. over various timescales, but the author chooses to leave this development until later in the book and to insert a section on geographical trends in species richness and other biotic features. If one reads the book sequentially, then this tends to disrupt a well-constructed argument - but the topic is one of considerable biological interest and certainly demands coverage. There are some biogeographical generalizations that are supra-taxonomic, such as climatic relationships to diversity, vegetation architecture, life form, leaf form and so on, that can assist in an understanding of the spatial arrangements of the world's biota. Hengeveld tackles this section of the book by choosing specific examples to illustrate the general development of biogeographical thought in these fields, such as gradients of bird richness in North America, species "nesting" and its relationship to refugia hypotheses, $\mathrm{C} 3 / \mathrm{C} 4$ photosynthetic strategies and climate, and many others.

The author finally returns to the theme of individual species' ranges and their analysis. He considers general features of population response curves to environmental variables and illustrates these with examples in which species boundaries can be related to climatic factors. On its own, however, such an approach might be regarded as static rather than dynamic, so Hengeveld concentrates on species for which there is adequate historical information from the fossil record so that the time factor can be incorporated into the model. The examples he uses, like Tilia cordata, are selected to illustrate the potential of this technique. But such relationships are rarely simple and neat, and in the final section of the book Hengeveld considers some of the complicating factors, such as the stochastic impact of weather and the lag that may exist between environmental change and species response.

The book provides a realistic account of what has been achieved by modern biogeography. But it also leaves me with the impression that the future of biogeographical development is largely in the hands of the ecophysiologist and the population geneticist, with some help from the statistician. I would say that the taxonomist and the palaeoecologist still have important parts to play in the assembling of data upon which testable hypotheses can be established.

This is a stimulating book, well written and arranged. There is a refreshing, logical line of argument that underlies the text and which leads the reader painlessly through the morass of information that typifies biogeography. It well justifies its claim to be dynamic.

Peter D. Moore is in the Division of Biosphere Sciences, King's College London, Campden Hill Road, London W8 7AH, UK. 